\title{
Grasping and hitting moving objects
}

\author{
Willemijn D. Schot • Eli Brenner · Jeroen B. J. Smeets
}

Received: 1 February 2011/Accepted: 30 May 2011/Published online: 11 June 2011

(C) The Author(s) 2011. This article is published with open access at Springerlink.com

\begin{abstract}
Some experimental evidence suggests that grasping should be regarded as independent control of the thumb and the index finger (digit control hypothesis). To investigate this further, we compared how the tips of the thumb and the index finger moved in space when grasping spheres to how they moved when they were hitting the sphere using only one digit. In order to make the tasks comparable, we designed the experiment in such a way that subjects contacted the spheres in about the same way in the hitting task as when grasping it. According to the digit control hypothesis, the two tasks should yield similar digit trajectories in space. People hit and grasped stationary and moving spheres. We compared the similarity of the digits' trajectories across the two tasks by evaluating the time courses of the paths of the average of the thumb and the index finger. These paths were more similar across tasks than across sphere motion, supporting the notion that grasping is not controlled fundamentally differently than hitting.
\end{abstract}

Keywords Prehension · Interception · Motor control

\section{Introduction}

In 1999, Smeets and Brenner hypothesized that the movements of the thumb and the index finger are

W. D. Schot $(\varangle) \cdot$ E. Brenner · J. B. J. Smeets

Research Institute MOVE,

Faculty of Human Movement Sciences,

VU University Amsterdam, Van der Boechorststraat 9,

1081 BT Amsterdam, The Netherlands

e-mail:w.d.schot@vu.nl controlled independently when grasping with a precision grip (Smeets and Brenner 1999). This was a break from the traditional hypothesis that reaching and grasping are controlled independently (Jeannerod 1984) and has led to much debate (e.g. Mon-Williams and McIntosh 2000; van de Kamp and Zaal 2007). The hypothesis of independent digit control assumes that the central nervous system primarily controls the positions of the end effectors in space. How the muscles are activated to achieve this and the postures this results in is a separate issue. Support for the idea that the positions of the end effectors in space are controlled come from studies that show that task-relevant degrees of freedom of a redundant system such as the human arm are selectively stabilized (e.g. Latash et al. 2002) and that there is less variability in the trajectories of the end effectors in a world-centred compared to a body-centred reference frame (Marteniuk and Bertram 2001).

The hypothesis of independent digit control leads to the prediction that if one can ensure that the constraints for a digit are equal when grasping and when performing a comparable single-digit task such as touching, pushing or hitting, the movements of that digit in space should be the same. Combining the paths of the thumb and the index finger when pushing with the thumb and index finger, respectively, should therefore look like a grasping movement. However, no matter how much effort one invests in making the two tasks as similar as possible, some constraints at the execution level will always differ. The most obvious example is that through the anatomical link between the digits, movements of the thumb and the index finger will influence each other in grasping. So, even for the same control of the positions of the thumb and the index finger in space in grasping and hitting, the paths will not be the same in the two tasks because there are 
inevitable differences between the tasks at the execution level (Smeets and Brenner 2001).

To compare the control of the digits' positions in space during grasping and hitting independently of differences between grasping and hitting that arise at the execution level as a result of the anatomical link between the thumb and the index finger, we used a measure that is not sensitive to the latter influence: the path of the average of the thumb and the index finger (Smeets et al. 2010), further referred to as the average path. Because we assume that the influence of the anatomical link between the thumb and the index finger is as large for the thumb as for the index finger and that their velocity profiles are similar, the average path should be the same for grasping as for hitting. If these assumptions are not correct, we may find differences between grasping and hitting that will incorrectly make us reject our hypothesis that grasping and hitting are controlled in the same way.

A problem with comparing the average paths of grasping and hitting is that it is not clear how small the differences between the paths have to be before the two tasks can be regarded as likely being controlled in the same way. We cannot fix a value for this, but we can test two predictions based on the reasoning in the previous paragraph. The first is that if we introduce some manipulation that is relevant to both tasks, such as changing the object's speed, the average paths may all change due to the new constraints imposed by the change in speed, but the difference between the average paths for the two tasks should not increase (unless the change in speed constrains the tasks differently). The second prediction is that any change to the target that is likely to be relevant to the task, and therefore clearly influences the constraints, should have at least as much influence on the average paths as changing the task between grasping and hitting.

In this article, three experiments will be described. In all three experiments, subjects grasped and hit the moving and stationary spheres to the left of their bodies with their right hand. In the first experiment, if the sphere was moving, it was moving from right to left at $1.00 \mathrm{~m} / \mathrm{s}$. The results showed that the average path was more similar for grasping and hitting than for movement towards stationary and moving targets. There were large differences between the velocity profiles of movements toward stationary and moving spheres. The effect of the target motion on the average paths was the same for grasping as for hitting. Two more experiments were conducted in which the effect of target motion on the hand velocity profiles was smaller. The effect of target motion on the average path was smaller than in the first experiment, but it never became smaller than the difference between grasping and hitting.

\section{Experiment 1}

\section{Methods}

Before the experiment started, subjects received information about the nature and duration of the experiment and signed an informed consent form. The experiment is part of an ongoing research programme that has been approved by the local ethics committee.

\section{Procedure}

Seven subjects (age 23-41, 2 men, all right-handed by selfreport) with no known neurological disorders made movements with their right hand towards a sphere that was either rolling from right to left at $1.00 \mathrm{~m} / \mathrm{s}$ or lying stationary at one of the three possible positions on a horizontal track (Fig. 1a). The track had a short ramp at the beginning on which a sphere could be placed. A release mechanism kept the sphere in place until the start of the measurement triggered it to release the sphere. The sphere accelerated on the ramp and the subjects grasped or hit the sphere well away from the ramp when the sphere was moving at nearconstant velocity. Separate measurements showed that the deceleration of a sphere initially moving at $1.00 \mathrm{~m} / \mathrm{s}$ on the horizontal part of the track was only $0.006 \mathrm{~m} / \mathrm{s}^{2}$.

A

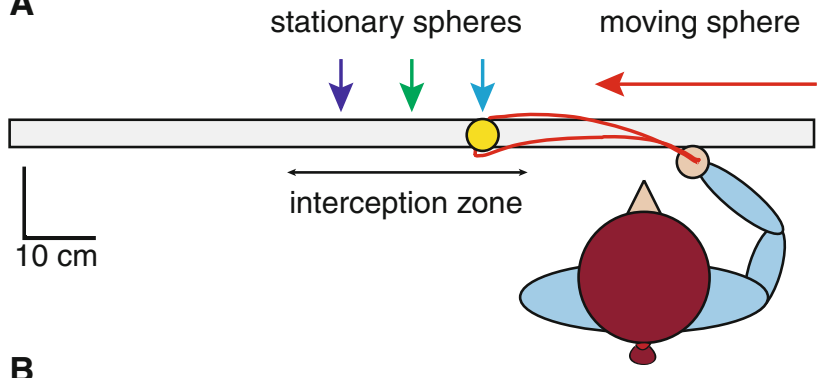

B

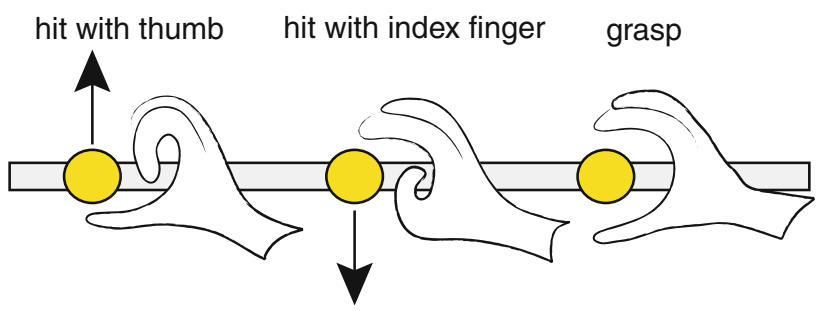

Fig. 1 Set-up and task a Top view of a single trial: the subject moved her hand from the starting position towards a sphere that was either stationary at one of three positions indicated by the three arrows or moving at a speed of $1 \mathrm{~m} / \mathrm{s}$ from right to left along the horizontal track. b Three different tasks: depending on the instruction given before the trial started, the subject hit the sphere away from him or herself with the thumb (left), hit it towards him or herself with the index finger (middle) or grasped it between thumb and index finger. All three tasks were performed with both stationary and moving spheres 
The task was to hit the sphere away from their bodies with their thumb, hit it towards their bodies with their index finger or grasp it between thumb and index finger (Fig. 1b). In the hitting tasks, the digit performing the task is referred to as the active digit. The other digit is referred to as the passive digit. The movements started with thumb and index finger touching each other at the starting position, $30 \mathrm{~cm}$ to the right of the rightmost sphere position (Fig. 1a). On trials in which the sphere was rolling, subjects were free to choose the point at which they hit or grasped the sphere, as long as it was to the left of their left knee. This ensured that the rolling spheres were hit or grasped at approximately the same positions as the stationary ones. The area in which subjects could hit or grasp the sphere will be referred to as the interception zone. Note that subjects could increase their viewing time by intercepting the sphere further to the left in the interception zone.

Movements were recorded at $250 \mathrm{~Hz}$ with an Optotrak motion recording system (Northern Digital, Waterloo, Ontario, Canada). Small clusters of three infrared emitting diodes (IREDs) were attached to the nails of the thumb and the index finger. A calibration trial was performed to be able to reconstruct the approximate positions of the contact points from the positions of the markers attached to the nails. During this trial, the subjects held an IRED between thumb and index finger such that the IRED was approximately at the position of the contact points of the thumb and the index finger with the sphere.

Another two markers were placed behind the track (10 and $20 \mathrm{~cm}$ to the right of the starting position). These markers were occluded for a few samples when the sphere rolled along the track. A separate measurement with a third marker on the other end of the track showed that there was no significant deceleration so linear extrapolation could be used to determine the position of the sphere rolling on the track at each moment during a trial (until it was hit or grasped).

Each subject performed 10 trials for each of the tasks (hit with thumb, hit with index finger and grasp) towards each of the three stationary positions and 20 trials for each of the tasks towards the rolling sphere. Each subject performed the 150 trials in fully randomized order.

\section{Data analysis}

Data were analysed offline using custom software. Movements were filtered using a second-order low-pass Butterworth filter with a cut-off frequency of $10 \mathrm{~Hz}$. We used the relationship between the clusters of three markers attached to the nails and the single marker held between finger and thumb during the calibration to determine the position of the contact points of the digits. The beginning of each movement was defined as the first moment the speed of the wrist exceeded $0.1 \mathrm{~m} / \mathrm{s}$. The end of the movement was determined using the MSI method that has been described in detail elsewhere (Schot et al. 2010). This method finds the moment that can best be considered to be the end of the movement. A full account of which criteria we used and how this was implemented is provided in the appendix. Movement time was defined as the time between movement onset and movement offset and was analysed with a Repeated Measures ANOVA with task (grasp, hit with thumb and hit with finger) and target motion (stationary, moving) as within-subject variables.

Velocity profiles were obtained by numerical differentiation of the position data. Peak velocity and time to peak velocity were analysed with Repeated Measures ANOVAs with task (grasp, hit), digit (thumb, finger) and target motion (stationary, moving) as within-subject variables.

We obtained four average paths for each subject: one for grasping a stationary target, one for grasping a moving target, one for hitting a stationary target and one for hitting a moving target. The latter two are the combination of the finger in hitting with the finger and the thumb in hitting with the thumb tasks. We used the average of the paths towards stationary targets at the three positions to better match the variability in the endpoints for stationary targets to that for moving targets, because there was substantial variability in the endpoints of movements to moving targets, both between and within subjects.

To obtain the average paths, we spaced a hundred points equally along the movement path of each digit. Within each subject, we averaged the digits' movement paths at these points. We then averaged the movement paths of the thumb and the index finger to obtain the average path for each condition. As is shown in Fig. 2, if the thumb (fat lines) moves out further in hitting (solid lines) than in grasping (dashed lines) by the same amount as the finger does, the averages for hitting and grasping will be the same.

We calculated the absolute difference between the average paths when grasping and hitting a moving target

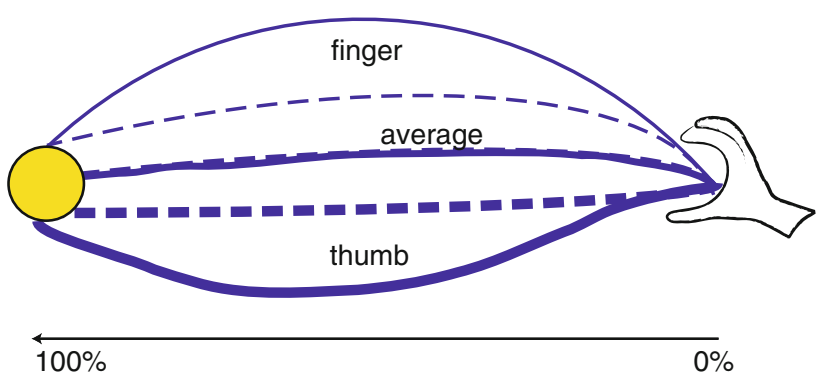

Fig. 2 Average path: To obtain the average path, we averaged the movement paths of the thumb and the index finger. We hypothesize that in hitting (solid lines) the thumb and the index finger might curve out more than in grasping (dashed lines) because in the former case, there is less influence of the tissue connecting the digits. However, because this influence is likely to be symmetrical, the average paths in grasping and hitting are predicted to be very similar 
(Gm-Hm), grasping and hitting a stationary target (Gs-Hs), grasping a stationary and a moving target (Gs-Gm), and hitting a stationary and a moving target (Hs-Hm). This difference between the average paths of two conditions was calculated for each subject by taking the mean of the absolute differences at each of the 100 points sampled along the curves. If our prediction that any relevant change to the target should have at least as much influence on the average paths as changing the task between grasping and hitting is true, the difference between the average paths of grasping and hitting should not be larger than the difference between the average paths of moving to a stationary and a moving target. We compared the average of $\mathrm{Gm}-\mathrm{Hm}$ and Gs-Hs (task different) with the average of Gs-Gm and Hs-Hm (target different) using a one-tailed paired $t$ test to evaluate this. If this $t$ test is significant, it indicates that movements towards a moving and a stationary target are more similar than grasping and hitting are, forcing us to reject our hypothesis.

If our prediction that target motion influences grasping in the same way as it influences hitting is true, the difference between the average paths for grasping and hitting should be the same regardless of whether the target is moving. Put differently, the difference between the average paths for movements towards stationary and moving targets should be the same regardless of whether the movement is grasping or hitting. That means that there should be no difference between Gm-Hm and Gs-Hs and between Gs-Gm and Hs-Hm. This was tested using twotailed paired $t$ tests.

It has previously been shown that the shapes of the average paths when tapping or grasping a stationary target vary more strongly between subjects than within subjects but between tasks (Smeets et al. 2010). To assess the differences between the average paths of the different subjects in the experiments presented here, we calculated the difference between each subject's average path and that of each other subject for every condition and averaged these differences across pairs of subjects and across conditions. We will use this measure as an indication of the resemblance between average paths when controlled independently for the same task.

\section{Results}

The average trajectories of the thumb and the index finger are shown in Fig. 3. As expected, trajectories of hitting movements are slightly wider than those of grasping movements. Also, target motion seems to affect the trajectories. The movement times, peak velocities and the times to peak velocity are shown in Fig. 4. On average, subjects took $572 \pm 42 \mathrm{~ms}$ to complete the movements. A Repeated Measures ANOVA with movement time as the dependent variable and task (grasp, hit with thumb and hit with index) and target motion (stationary, moving) as within-subject variables showed that there were differences between the tasks $(F(2,12)=22.63, P<.001)$. Pairwise comparisons revealed that grasping was performed more slowly than hitting with either the thumb $(P=.001)$ or the index finger $(P=.011)$. There was also a significant task by movement interaction $(F(2,12)=9.74, P=.003)$, but none of the post hoc tests reached significance.

The average velocity profiles in the main direction of motion are shown in Fig. 5. The average peak velocity was $1.19 \pm 0.04 \mathrm{~m} / \mathrm{s}$. A Repeated Measures ANOVA with task (grasp, hit), target motion (stationary, moving) and digit (thumb, index) as within-subject variables revealed that the peak velocity in grasping was lower than in hitting $(F(1,6)=14.45, P=.009)$. The peak velocity was higher for the index finger than for the thumb $(F(1,6)=19.67$,
Fig. 3 Top view of the trajectories of the thumb and the index finger, averaged over subjects

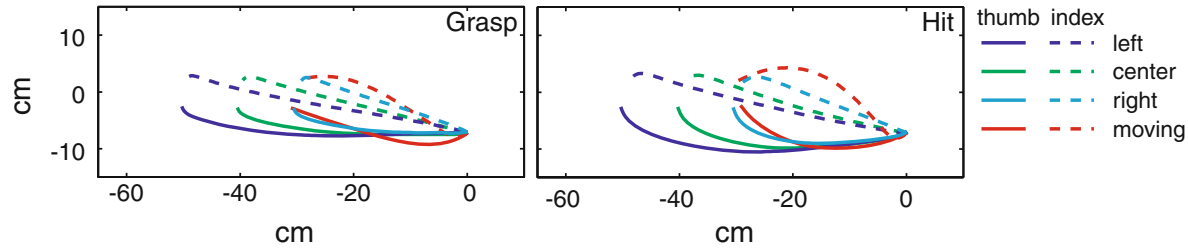

MT
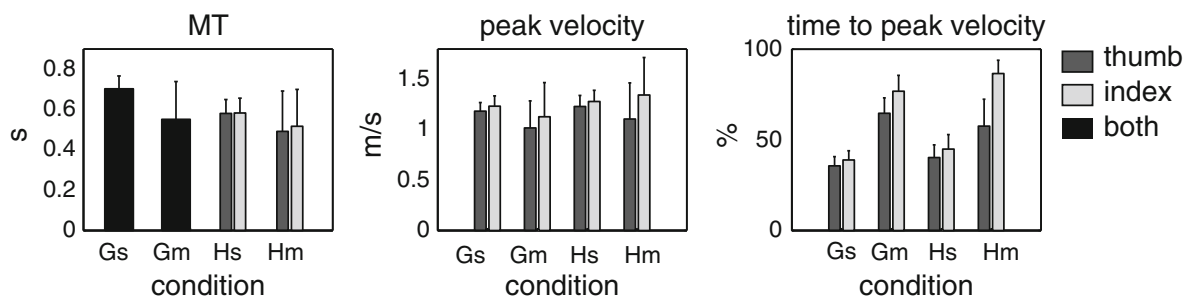

Fig. 4 Movement time (left), peak velocity (middle) and time to peak velocity as a percentage of movement time (right) for grasping the stationary sphere $(G s)$, grasping the moving sphere $(G m)$, hitting the stationary sphere $(H s)$ and hitting the moving sphere $(H m)$ 


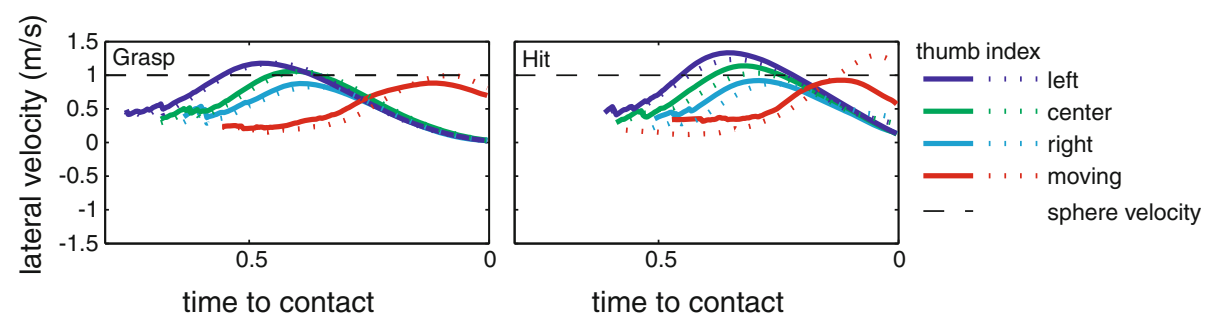

Fig. 5 Lateral velocity as a function of time to contact. Data are the averages per subject averaged over subjects. Because we align the data on time to contact and there is variability in the movement times both within and between subject, the beginning of the average velocity is not very reliable. We only show the average from the moment that at least 2 subjects have performed at least 5 trials. Target motion clearly shifts the time of the peak velocity further to the end of the movement
$P=.004)$. The difference between stationary and moving targets was $0.03 \mathrm{~m} / \mathrm{s}$ larger for the thumb than for the index finger (digit by target movement interaction: $F(1,6)=$ 12.66, $P=.012)$.

On average, the peak velocity occurred at $55.7 \pm 2.5 \%$ of the movement time. A Repeated Measures ANOVA with task (grasp, hit), target motion (stationary, moving) and digit (thumb, index) as within-subject variables showed that it occurred much earlier in the movements towards stationary spheres $(39.9 \pm 2.2 \%)$ than in movements towards moving spheres $(71.40 \pm 2.9 \%, F(1,6)=393.81, P<.001)$. It also occurred earlier in the thumb than in the index finger $(F(1,6)=55.99, P<.001)$. The difference in the time of the peak velocity between hitting and grasping was larger for the index finger than for the thumb $(F(1,6)=12.96, P=.011)$. This was also the case for the difference between movements towards moving and stationary spheres $(F(1,6)=15.16$, $P=.008)$. There was also a significant task by digit by target motion interaction $(F(1,6)=12.66 . P=.012)$.

The significant differences mentioned above cannot distinguish between the various hypotheses about such movements are controlled because they could just reflect differences in constraints for the different tasks. That is why we based our predictions on the average paths. As predicted, the difference between the average paths when comparing hitting with grasping is not larger than the difference when comparing moving towards a stationary with moving towards a moving target $(t(6)=-3.21, P=.991)$. We only tested whether the difference between the tasks is larger than the difference between the target motions because this is the critical comparison. However, it is clear from Fig. 6 that the difference between the tasks is smaller than the difference between the target motions. It is also clear that the shapes of the average paths differ between subjects. The mean difference between the individual subjects' average paths (open bar in Fig. 6) is larger than the differences between the tasks.

The effect of target motion on time to peak velocity is the same for grasping as for hitting (task by target motion: $F(1,6)=0.35, P=.109)$ so we expect the effect of target motion on the average paths to be the same for grasping as for hitting. This is indeed the case: $(t(6)=-1.87, P=.111$ and $t(6)=0.33, P=.753$ for $\mathrm{Gm}-\mathrm{Hm}$ versus Gs-Hs and GsGm versus Hs-Hm, respectively, see Fig. 6).

\section{Discussion}

In this experiment, we examined whether the differences in the average paths of the digits between grasping and hitting are no larger than those between movements towards moving and stationary targets. We found that, in agreement with our hypothesis, the differences between grasping and hitting were smaller than the differences between movements towards stationary and moving targets. The effect of target motion on the average paths was the same for grasping as for hitting.

Because the target was moving from right to left and the hand also had to move from right to left, subjects were inclined to move along with the moving targets when they intercept them, resulting in large differences in the lateral velocity profiles between movements towards stationary and moving targets. If the lateral velocity profile is affected differently by the sphere's motion than the tangential velocity profile, the average path will be affected as well. Therefore, if we can arrange for the lateral velocity profiles to be affected less by target motion, the average paths of movements towards stationary targets will be more similar to the average paths of movements towards moving targets, providing a stronger test for our hypothesis. In the second experiment, we examine whether decreasing the difference between the velocity profiles will make the difference between the average paths of movements towards stationary and moving targets become smaller than the difference between grasping and hitting. If it does, we will have to reject our hypothesis.

\section{Experiment 2}

Seven different subjects (age 23-28, 3 men, 6 right-handed and 1 ambidextrous by self-report) with no known 

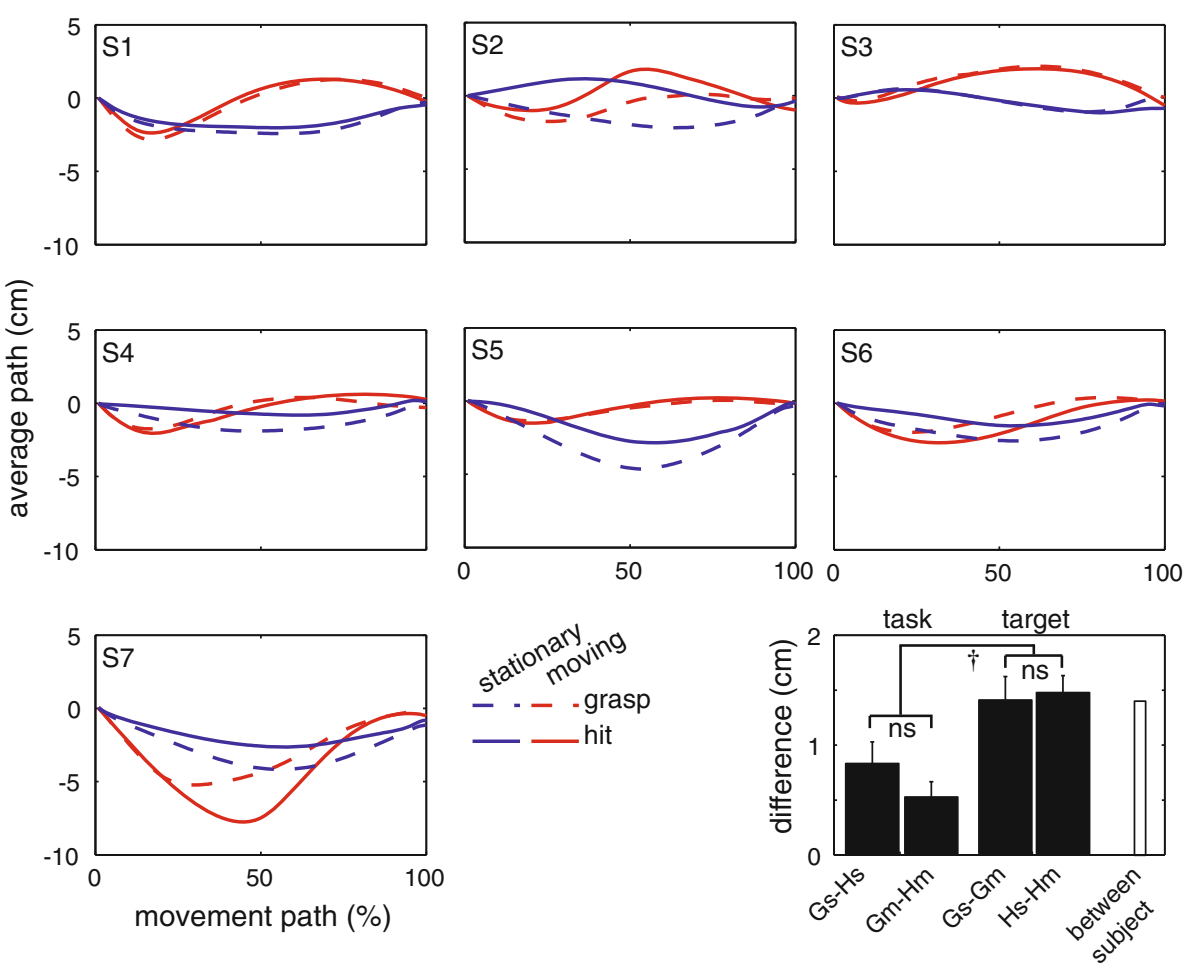

Fig. 6 Average paths per condition for each subject. The average of the thumb and finger is plotted as a function of the percentage of the total movement path. If the thumb curves out further than the index finger, this value is negative. The bar graph shows the average (mean $\pm \mathrm{SE}$ ) absolute differences between the conditions (differences between the curves), ${ }^{\dagger}=$ one-sided $t$ test not significant (the large difference is in the opposite direction of the hypothesis), $n s=$ two-sided $t$ test not significant. As hypothesized, moving

neurological disorders participated in an experiment that was identical to Experiment 1 with the exception that the target now moved from left to right. This means that the target and the subject's hand are moving towards each other rather than in the same direction. We expect this to make the velocity profiles of movements towards stationary spheres more similar to the velocity profiles of movements towards moving spheres because if the hand is moving in the opposite direction than the sphere, it is less advantageous to move along with the moving sphere at the time of the grasp or hit. Again, our hypothesis is that the differences between the average paths for grasping and hitting should be no larger than the differences between movements towards a moving or a stationary target. Moreover, the effect of target motion on the average paths should be similar for grasping and hitting.

Results

The average trajectories of the thumb and the index finger are shown in Fig. 7. A Repeated Measures ANOVA with task (grasp, hit), target motion (stationary, towards a moving and a stationary target are not more similar than grasping and hitting (on the contrary, the left pair of bars is lower than the right pair suggesting that grasping and hitting are more similar than moving towards a moving and a stationary target) and the effect of target motion the average paths is similar for grasping as for hitting (the heights of the right two bars do not differ significantly). The mean difference between the individual subjects' average paths is shown as an open bar

moving) and digit (thumb, index) showed that the peak velocity still occurred earlier in movements towards stationary targets than in movements towards moving targets $(F(1,6)=12.61, P<.012$, Fig. 8). However, in this experiment, the effect was much smaller. Peak velocity occurred at $45.1 \pm 2.5 \%$ when moving towards moving targets and $41.9 \pm 1.3 \%$ when moving towards stationary targets.

Now that the velocity profiles of movements towards stationary and moving targets are more similar, the difference between the average paths when comparing hitting with grasping is as large as the difference when comparing moving towards stationary with moving towards moving targets $(t(6)=-0.89, P=.796$, see Fig. 9). As in experiment 1 , the effect of target motion on time to peak velocity is the same for grasping as for hitting (task by target motion: $F(1,6)=0.64, P=.454$ ) so we expect the effect of target motion on the average paths to be the same for grasping as for hitting. This is indeed the case: $(t(6)=-0.78, P=.466$ and $t(6)=-0.76, P=.475$ for $\mathrm{Gm}-\mathrm{Hm}$ versus Gs-Hs and Gs-Gm versus Hs-Hm, respectively, see Fig. 9). 
Fig. 7 Top view of the trajectories of the thumb and the index finger averaged over subjects
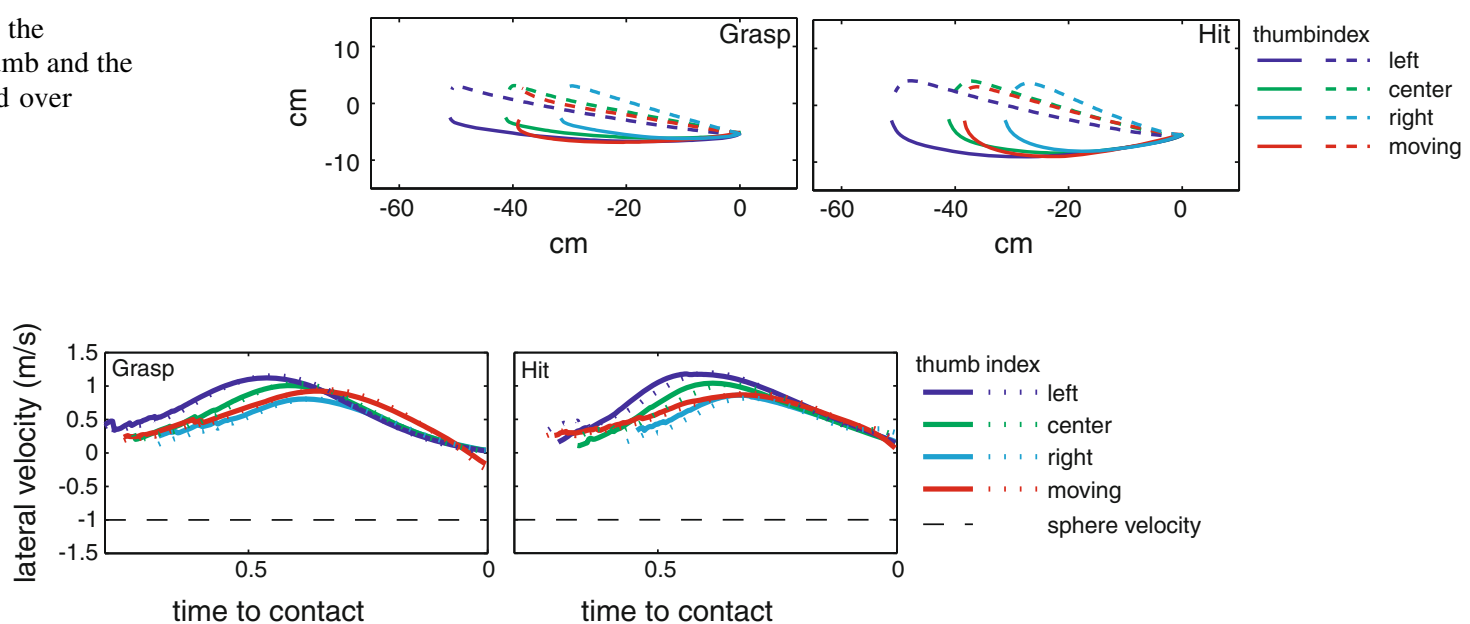

Fig. 8 Lateral velocity as function of time to contact. See Fig. 5 for further details. Reversing the direction of the sphere's motion compared to Experiment 1 (from left to right rather than from right to left) reduced the difference between the velocity profiles for static and moving spheres

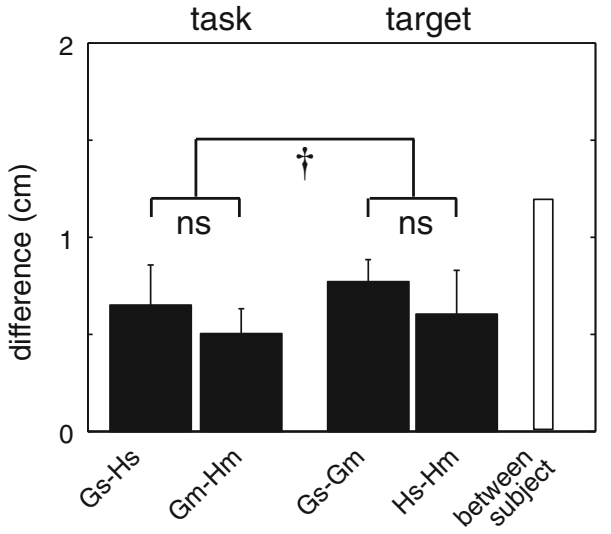

Fig. 9 Average differences between the conditions. Grasping and hitting differ as much from each other as moving towards a moving and a stationary target, and the effect of target motion the average paths is the same for grasping as for hitting. Values shown are means $\pm \mathrm{SE},{ }^{\dagger}=$ one-sided $t$ test not significant, $n s=$ two-sided $t$ test not significant. The mean difference between the individual subjects' average paths is shown as an open bar

\section{Discussion}

Reversing the direction of the moving sphere greatly reduced the difference in average paths between the movements towards stationary and moving spheres. We attribute this to the fact that the velocity profiles of movements towards moving spheres were now more similar to those towards stationary spheres. However, the differences between the average paths of grasping and hitting movements were still not larger than those between movements towards stationary and moving spheres. In the third experiment, we wanted to test whether making the difference between stationary and moving spheres smaller, by reducing the speed of the moving spheres, would also make the difference between the average paths of movements towards stationary and moving spheres smaller, forcing us to reject our hypothesis.

\section{Experiment 3}

Seven new subjects (age 23-30, 1 man, 6 right-handed and 1 ambidextrous by self-report) with no known neurological disorders performed an experiment that was identical to the second experiment with the exception that the speed of the sphere on the moving target trials was reduced to $0.56 \mathrm{~m} / \mathrm{s}$. The deceleration of a sphere moving at this initial speed was about $0.02 \mathrm{~m} / \mathrm{s}^{2}$.

\section{Results}

The average movement paths per condition are shown in Fig. 10. Reducing the speed of the moving sphere did not have a large effect on the time to peak velocity (Compare Fig. 11 with Fig. 8). A Repeated Measures ANOVA with task (grasp, hit), target motion (stationary, moving) and digit (thumb, index) showed that the peak velocity still occurred earlier in movements towards stationary targets than in movements towards moving targets $(F(1,6)=$ $12.19 P<.013$, Fig. 11). Peak velocity occurred at $42.7 \pm 2.0 \%$ for moving targets and $38.2 \pm 1.8 \%$ for stationary targets.

The difference between the average paths of movements toward a moving and a stationary target is still not smaller than the difference between grasping and hitting $(t(6)=$ $-0.75, P=.760$, see Fig. 12). It is even slightly larger. The effect of target motion on time to peak velocity is, however, not the same for grasping as for hitting (task by target motion: $F(1,6)=39.58, P=.001$ ), so it is not clear whether, according to our hypothesis, the effect of target 
Fig. 10 Top view of the trajectories of the thumb and the index finger, averaged over subjects

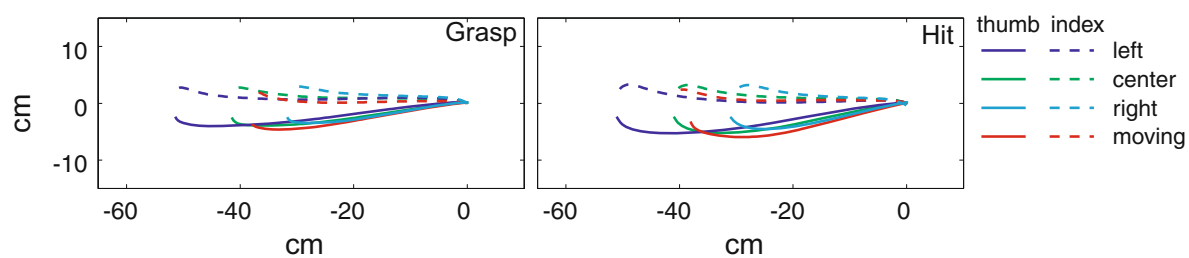

Fig. 11 Lateral velocity as a function of time to contact. See Fig. 5 for further details
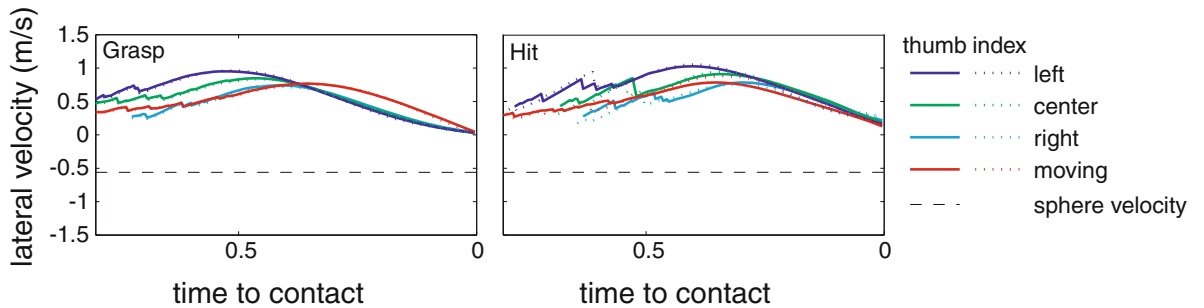

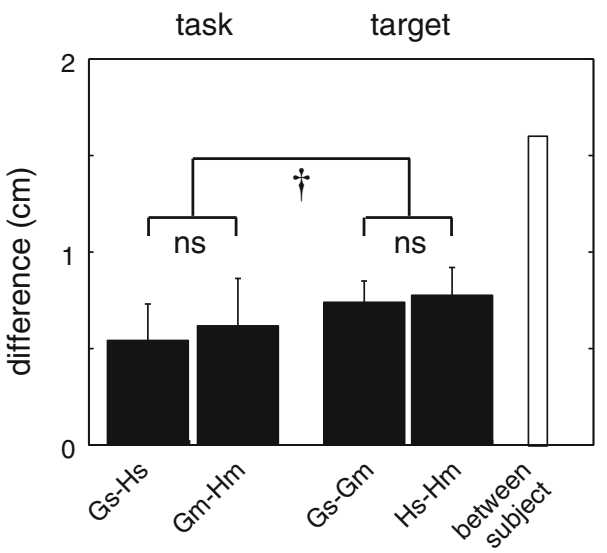

Fig. 12 Average differences between the conditions. Grasping and hitting differ as much from each other as moving towards a moving and a stationary target and the effect of target motion on the average paths is the same for grasping as for hitting. Values shown are means $\pm \mathrm{SE},{ }^{\dagger}=$ one-sided $t$ test not significant, $n s=$ two-sided $t$ test not significant. The mean difference between the individual subjects' average paths is shown as an open bar

motion on the average paths should be the same for grasping as for hitting. The results show that the effect of target motion on the average paths is still the same for grasping as for hitting: $(t(6)=0.84, P=.431$ and $t(6)=0.40, P=.703$ for $\mathrm{Gm}-\mathrm{Hm}$ versus Gs-Hs and GsGm versus Hs-Hm, respectively, see Fig. 12).

\section{General discussion}

We wanted to study whether grasping and hitting are controlled in the same way. There were three issues we faced when trying to test this idea. We posed that, if grasping and hitting are controlled in the same way and all other task constraints are the same, the paths of the digits would be the same for the two tasks. The first issue was that task constraints are never exactly the same. We solved this for one evident difference in constraints (the influence of the tissue connecting the thumb and the index finger) by looking at the average path of the thumb and the index finger.

The second issue was that we did not know how small the differences between grasping and hitting have to be before we can regard them as being controlled in the same way. We chose to use a manipulation that would affect both grasping and hitting. We hypothesized that grasping and hitting are controlled in the same way; therefore, affecting them both should not lead to differences between grasping and hitting that are larger than the differences caused by the manipulation.

The manipulation that we used to test these predictions is object motion. It has been proposed that movements towards stationary and moving objects are controlled in the same way (Zaal et al. 1999; Dessing et al. 2002), although the hand's trajectories are different when hitting moving targets than when hitting stationary ones (Brenner and Smeets 2007). The former made it unlikely that the differences due to the manipulation (object motion) are so fundamental that they completely mask any other differences, whereas the latter made sure that some differences will be obtained.

We performed three experiments to test the differences in the average path between grasping and hitting targets and between moving towards moving and stationary targets. In all cases, the difference between grasping and hitting was either smaller than or as large as the difference between moving towards a moving or a stationary target. Also, the effect of target motion on the average paths was always the same for grasping as for hitting.

Implications for control

The third issue is that our reasoning only holds one way. That is, if the control and the task constraints are the same, the paths should be the same; but if the paths are the same, 
this does not necessarily mean that the control is the same. Although the a priori probability is very small, different control strategies can, in principle, lead to the same movement paths (Smeets and Brenner 1999). More explicitly, it could be that coincidentally, the average path of the hitting movements is the same as the average path of the grasping movements even though the underlying control mechanism is different. The theory that grasping should be considered as a transport and a grip component makes no claims about hitting. Importantly, it cannot make any claims about hitting because the grip component needs both digits and the transport is of the wrist rather than the digits. Therefore, in this view, grasping and hitting should be considered as independent and any similarities between grasping and hitting, such as the ones reported here, should be considered a coincidence.

If the individual subjects' average paths would never change, we would have been able to attribute them to anatomical or postural constraints, but since we see clear differences between the paths towards static and moving targets, the paths must be considered to at least partly arise from the control strategy. Thus, to account for the current data, the above-mentioned coincidence would have to occur for the majority of the subjects individually because individual subjects clearly tend to have their own characteristic movement pattern (the difference between the individual subjects' average paths was $1.2-1.6 \mathrm{~cm}$, which is clearly larger than the differences between grasping and hitting; Figs. 6, 9, 12). Thus, our results suggest that hitting and grasping are controlled in the same way.

Common control for grasping and hitting would not necessarily imply that both movements are performed in the same manner. Besides control, there are at least two elements that can influence the movements. First, subjects have to translate the task (grasp or hit) into movement constraints. They could adopt different strategies for the different tasks. For instance, they might prefer to have a different final lateral velocity when grasping than when hitting. Given this, it is striking that the velocity profiles for hitting and grasping are so similar and that they are affected by target motion in nearly the same way.

Second, the movements of the digits in space have to be accomplished by changing joint angles. Except for the fact that the subjects had to stay in their seat, they were free to move any way they liked as long as they made a fluent movement to the target. It is possible that the movements they made with, for instance, their hip, shoulder, elbow, wrist and phalangeal joints are very different. However, this does not argue against common control. On the contrary, if the similarity between grasping and hitting in the average paths has arisen even though the movements of the joints were very different, it would add to the idea that it is the positions of the endpoints that is primarily controlled during grasping and hitting. This is exactly the level at which we proposed grasping and hitting to be equivalent.

Altogether, we do not provide conclusive evidence that the digits (rather than reach and grasp components) are controlled independently. However, we do show that it is unnecessary to assume a different control mechanism for grasping and hitting. An advantage of common control of hitting and grasping is that you need only two controllers (one for the thumb and one for the index finger) to perform grasping as well as hitting movements. In a view where reaching and grasping are controlled independently, one would need four controllers (reach and grasp for grasping and finger and thumb for hitting). Moreover, the similarities and differences between the average paths are consistent with predictions based on similar control of the digits for grasping and hitting, whereas this pattern would require additional argumentation to be reconciled with the control of reach, grasp, finger hit and thumb hit.

Acknowledgments This research was funded by NWO/MaGW under grant number 400-03-021.

Open Access This article is distributed under the terms of the Creative Commons Attribution Noncommercial License which permits any noncommercial use, distribution, and reproduction in any medium, provided the original author(s) and source are credited.

\section{Appendix}

The end of the movement was determined by using the MSI method (Schot et al. 2010). This entails multiplying several functions describing various criteria describing the end of the movement. First we will describe six binary functions. These functions have a value of 1 when a criterion was met and 0 when it was not.

(1) For all trials, the lateral position of the average of the thumb and the index finger had to be between 12.5 and $65.0 \mathrm{~cm}$ left of the starting position and (2) the sphere and the active digit (in grasping both digits) should not be further than $4 \mathrm{~cm}$ apart. (3) The active digit (in grasping, both digits) had to be between $2.0 \mathrm{~cm}$ below and $4.5 \mathrm{~cm}$ above its starting position, and (4) it should be on the appropriate side of the track. (5) Because the spheres were always to the left of the starting position, movements from left to right were assumed only to occur from just before to well after the end of the movement we were interested in. Therefore, time points more than $200 \mathrm{~ms}$ after the first rightward movement were considered too late to be the end of the movement.

For the trials in which the sphere was stationary, an additional binary requirement was that (6) the sagittal velocity of the active digit should be in the correct 
direction. That is, the thumb should be moving away from the body and the index finger towards the body. We did not require this for the trials in which the sphere was moving because on those trials, the task could also be achieved by letting the sphere hit the fingers. In that case, the sphere could be pushing the fingers apart, making the abovementioned requirement invalid.

In addition to the binary criteria, we also used two continuous functions to describe the likelihood that certain time points are the end of the movement. (7) We required the lateral position of the digits to be close to the lateral position of the sphere. If the sphere was rolling, the lateral position of the sphere is calculated using information from the two markers behind the track about when these markers were occluded by the sphere. The criterion is implemented in Eq. 1 where $\Delta b$ denotes the absolute difference in lateral position of the active digit and the lateral position of the sphere. $\Delta b$ can range from 0 to $\Delta b_{\max }$. In case the velocity of the sphere was misjudged on some trials, we set $F_{7}$ to 0.5 if $\Delta b$ was larger than $2.25 \mathrm{~cm}$. In the case of grasping, $F_{7}$ was calculated for the thumb and the index finger and both were entered in $F_{\text {total }}$.

$F_{7}(\Delta b<2.25)=1-\frac{\Delta b}{\Delta b_{\max }}$

$F_{7}(\Delta b>2.25)=0.5$

(8) We also required the sagittal position of the active digit to be close to the sagittal position of the sphere. As the centre of the sphere was at 0 , the thumb was expected to be close to -2.25 and the index finger close to 2.25 at the end of the movement. This is implemented in Eq. 2 where $\Delta l$ denotes the absolute deviation from -2.25 or 2.25 depending on whether the function is applied to, respectively, the thumb or the index finger. Again, in the case of grasping, the function was calculated for both digits.

$$
F_{8}\left(\text { sagpos }_{\text {thumb }}<0 \& \text { sagpos }_{\text {index }}>0\right)=1-\frac{\Delta l}{\Delta l_{\max }}
$$

The endpoint of a trial is the maximum of $F_{\text {total }}$ given in Eq. 3.

$F_{\text {total }}=\prod_{i=1}^{8} F_{i}$

\section{References}

Brenner E, Smeets JBJ (2007) Flexibility in intercepting moving objects. J Vis 7:1411-1417

Dessing JC, Bullock D, Peper CL, Beek PJ (2002) Prospective control of manual interceptive actions: comparative simulations of extant and new model constructs. Neural Netw 15:163-179

Jeannerod M (1984) The timing of natural prehension movements. J Motor Behavior 16:235-254

Latash ML, Scholz JP, Schoner G (2002) Motor control strategies revealed in the structure of motor variability. Exerc Sport Sci Rev 30:26-31

Marteniuk RG, Bertram CP (2001) Contributions of gait and trunk movements to prehension: perspectives from world- and bodycentered coordinates. Mot Control 5:151-165

Mon-Williams M, McIntosh RD (2000) A test between two hypotheses and a possible third way for the control of prehension. Exp Brain Res 134:268-273

Schot WD, Brenner E, Smeets JBJ (2010) Robust movement segmentation by combining multiple sources of information. J Neurosci Methods 187:147-155

Smeets JBJ, Brenner E (1999) A new view on grasping. Mot Control 3:237-271

Smeets JBJ, Brenner E (2001) Independent movements of the digits in grasping. Exp Brain Res 139:92-100

Smeets JBJ, Martin J, Brenner E (2010) Similarities between digits' movements in grasping, touching and pushing. Exp Brain Res 203:339-346

van de Kamp C, Zaal FT (2007) Prehension is really reaching and grasping. Exp Brain Res 182:27-34

Zaal FTJM, Bootsma RJ, van Wieringen PCW (1999) Dynamics of reaching for stationary and moving objects: data and model. J Exp Psychol 25:149-161 